ARTICLE

https://doi.org/10.1038/s41467-019-10657-4

\title{
Spectral phase control of interfering chirped pulses for high-energy narrowband terahertz generation
}

Spencer W. Jolly (1) 1,2,7, Nicholas H. Matlis ${ }^{3,7}$, Frederike Ahr ${ }^{3}$, Vincent Leroux (1) 1,2, Timo Eichner ${ }^{1}$, Anne-Laure Calendron (10 3,4, Hideki Ishizuki ${ }^{5,6}$, Takunori Taira ${ }^{5,6}$, Franz X. Kärtner (1) ${ }^{3,4}$ \& Andreas R. Maier (1) ${ }^{1}$

Highly-efficient optical generation of narrowband terahertz radiation enables unexplored technologies and sciences from compact electron acceleration to charge manipulation in solids. State-of-the-art conversion efficiencies are currently achieved using differencefrequency generation driven by temporal beating of chirped pulses but remain, however, far lower than desired or predicted. Here we show that high-order spectral phase fundamentally limits the efficiency of narrowband difference-frequency generation using chirped-pulse beating and resolve this limitation by introducing a novel technique based on tuning the relative spectral phase of the pulses. For optical terahertz generation, we demonstrate a 13-fold enhancement in conversion efficiency for 1\%-bandwidth, $0.361 \mathrm{THz}$ pulses, yielding a record energy of $0.6 \mathrm{~mJ}$ and exceeding previous optically-generated energies by over an order of magnitude. Our results prove the feasibility of millijoule-scale applications like terahertz-based electron accelerators and light sources and solve the long-standing problem of temporal irregularities in the pulse trains generated by interfering chirped pulses.

\footnotetext{
${ }^{1}$ Center for Free-Electron Laser Science and Department of Physics Universität Hamburg, Luruper Chaussee 149, 22761 Hamburg, Germany. ${ }^{2}$ Institute of Physics of the ASCR, ELI-Beamlines project, Na Slovance 2, 18221 Prague, Czech Republic. ${ }^{3}$ Center for Free-Electron Laser Science and Deutsches Elektronen Synchrotron (DESY), Notkestraße 85, 22607 Hamburg, Germany. ${ }^{4}$ Department of Physics and The Hamburg Centre for Ultrafast Imaging, Universität Hamburg, Luruper Chaussee 149, 22761 Hamburg, Germany. ${ }^{5}$ Division of Research Innovation and Collaboration, Institute for Molecular Science, 38 Nishigonaka, Myodaiji, Okazaki, Aichi 444-8585, Japan. ${ }^{6}$ Innovative Light Sources Division, RIKEN SPring-8 Center, 1-1-1 Kouto, Sayo-cha, Sayo-gun, Hyogo 679-5148, Japan. ${ }^{7}$ These authors contributed equally: Spencer W. Jolly, Nicholas H. Matlis. Correspondence and requests for materials should be addressed to S.W.J. (email: spencer.jolly@cea.fr)
} 
ecent years have seen a tremendous surge in development of laser-based, high-field terahertz $(\mathrm{THz})$ sources for a wide range of applications, including $\mathrm{THz}$-based accelerators ${ }^{1-3}$, control and metrology of electrons ${ }^{4}$, femtosecond X-ray pulse characterisation $^{5,6}$, control of dynamics in solids ${ }^{7,8}$ and spectroscopy 9 . Most work has concentrated on generation of single-cycle, broadband $\mathrm{THz}$ pulses, resulting in a mature technology yielding close to millijoule energies and optical-to- $\mathrm{THz}$ conversion efficiencies near $1 \%^{10-13}$. Some emerging applications, however, such as waveguide-based electron acceleration ${ }^{14}$, resonant pumping of materials ${ }^{15}$ and narrowband spectroscopy ${ }^{9}$, require high-field, narrow-bandwidth $\mathrm{THz}$ pulses for which the technology is much less developed. In particular, relativistic acceleration of electrons calls for optically synchronised multimillijoule pulses with $\mathrm{MV} / \mathrm{cm}$ electric-field strengths, frequencies below $1 \mathrm{THz}$ and bandwidths below $1 \%{ }^{16,17}$, for which no technology currently exists.

Early on, periodically-poled lithium niobate (PPLN) was identified as promising for optical difference-frequency generation (DFG) of multicycle $\mathrm{THz}$ pulses ${ }^{18,19}$ due to its high nonlinear coefficient, $d_{33} \sim 25 \mathrm{pm} / \mathrm{V}$, and predicted conversion efficiencies in the range of several percent ${ }^{17}$. Such crystals would enable generation of $\mathrm{THz}$ pulses with tens-of-millijoule energies, sub-percent bandwidths and $\mathrm{MV} / \mathrm{cm}$ fields using the Joule-level optical pulses available today. However, the insufficient optimisation of the narrowband optical-to- $\mathrm{THz}$ conversion, and the resultant low fraction of optical photons that contribute to this process, is a primary obstacle to achieving $\mathrm{THz}$ pulses with the above parameters. Recent work has therefore concentrated on addressing the systematic optimisation of laser and crystal parameters with a view towards making every photon count.

Initial experiments using compressed Ti:Sapphire pulses demonstrated that the $\mathrm{THz}$ yield can be significantly increased $(\times 5)$ by optimising the optical bandwidth of the driver laser and by cryogenic cooling of the PPLN which reduces $\mathrm{THz}$ reabsorption $^{20}$. Using short-pulse drivers, however, results in high intensities that limit the energy that can be applied to the crystal before the conversion efficiency saturates or the crystal damages. In addition, the simultaneous presence of photons spanning a broad bandwidth enables phase-matched generation of unwanted, higher-frequency $\mathrm{THz}^{20}$, which not only reduces efficiencies by robbing energy from the main process, but also contributes to damage and thus reduces the total amount of useful pump energy that can be applied to the crystal.

These issues, however, can be overcome by pumping a nonlinear crystal with temporally chirped pulses. Chirping the drive pulse reduces the peak intensity, which allows for large increases in optical energy, and also reduces the instantaneous spectral bandwidth by mapping frequency onto time. In order to provide the correct spectral content for DFG, two chirped pulses must then be combined with a delay.

This chirp-and-delay technique, pioneered originally for driving photoconducting antennae ${ }^{21}$, has since been extensively used in a broad range of scientific settings ${ }^{22-24}$ because it provides a simple means of creating optical pulse trains of tunable number and periodicity which can be effectively used to drive resonant processes. A complication is that chirped pulses from chirpedpulse amplification (CPA) based laser systems inherently contain high-order phase, such as third-order dispersion (TOD), which induces temporal variations in the periodicity of the pulse train (temporal beating) and can reduce effectiveness of the driver ${ }^{23,25}$. For narrowband $\mathrm{THz}$ generation, the TOD-induced pulse-train periodicity variations, which are connected to temporal variations in the instantaneous difference-frequency content, can severely limit the conversion efficiency. Unfortunately, the intuition to completely remove the TOD from the chirped drive pulses leads to approaches which are either practically infeasible or produce undesired changes in the pulse properties.

Here, we present an elegant and easy-to-implement method for precisely controlling the temporal variation of the optical modulation period. Instead of removing the TOD, we simply tune the relative spectral phase between the chirped pulses. The former linearises the chirp, but requires an impractically large change to both pulses, while the latter uses a very small difference in chirp between the pulses to monochromatise the difference-frequency spectrum and regularise the pulse train. Our method not only enables significant optimisation for efficiently driving narrowband resonant processes but also offers new possibilities in producing designer pulse trains with varying periodicities optimised for specific purposes. We apply this technique to the problem of optimising the conversion efficiency for narrowband, multicycle $\mathrm{THz}$ generation by regularising the driver pulse train. By tuning the relative spectral phase in a chirp-and-delay setup, we achieve a 13-fold relative enhancement in conversion efficiency resulting in sub-percent bandwidth, $361 \mathrm{GHz}$ pulses with a conversion efficiency of $0.24 \%$. Using a large-aperture PPLN, we demonstrate a record energy of $0.6 \mathrm{~mJ}$ which exceeds previously reported energies by over an order of magnitude 26,27 .

\section{Results}

Monochromatising the difference-frequency spectrum. The idealised case for narrowband difference-frequency generation (DFG) calls for two narrow-line optical sources with a welldefined frequency difference, $\Delta \omega$. Unfortunately, these sources are not yet available with appreciable energies. Chirp-and-delay offers a way of obtaining, effectively, a two-line narrowband source from a broad-band, linearly chirped laser by combining two pulses with a delay $\Delta t$. The resulting instantaneous spectral features are narrow and have a constant frequency difference $\Delta \omega$. For this reason, chirp-and-delay is also known as spectral focusing ${ }^{28}$. By adjusting the delay, $\Delta \omega$ can be tuned to match the design frequency $\Omega_{\mathrm{THz}}$ of a PPLN crystal, enabling effective conversion of the provided difference-frequency content into a THz pulse (Fig. 1a).

A complication of this concept is that chirped pulses from CPA-based laser systems include not only group delay dispersion (GDD), i.e., linear chirp, but also non-negligible third-order dispersion (TOD) which adds a quadratic component, i.e., curvature, to the chirp (Fig. 1b). The result is a linear variation of the difference frequency with time (i.e., difference-frequency chirp) which detunes a fraction of the drive pulse energy away from the resonance condition (e.g., phase-matching) of the driven process. This effect becomes increasingly severe as the bandwidth of the resonance narrows, such as for difference frequency generation of narrowband $\mathrm{THz}$ pulses with a large number of cycles.

An intuitive solution would be to completely remove the TOD, thereby linearising the chirp. Unfortunately, this solution is generally impractical because most dispersive systems couple TOD with large amounts of GDD and thereby also produce undesired changes to the pulse duration. Although chirped mirrors can be designed to provide pure TOD, they typically compensate only of order $10^{4} \mathrm{fs}^{3}$ per bounce, while CPA systems usually have at least $10^{6} \mathrm{fs}^{3}$ of TOD. It is, however, not necessary to completely remove the TOD. What is actually important is to monochromatise the difference frequency, $\Delta \omega$, which can be achieved much more elegantly by simply adding a small amount of spectral phase to one of the two pulses (see Methods). The effect is to slightly tilt one pulse in time-frequency space (Fig. 1c) so that the temporal dependence of $\Delta \omega$ is eliminated despite the chirp curvature. In this way, $\Delta \omega$ remains within the narrow 

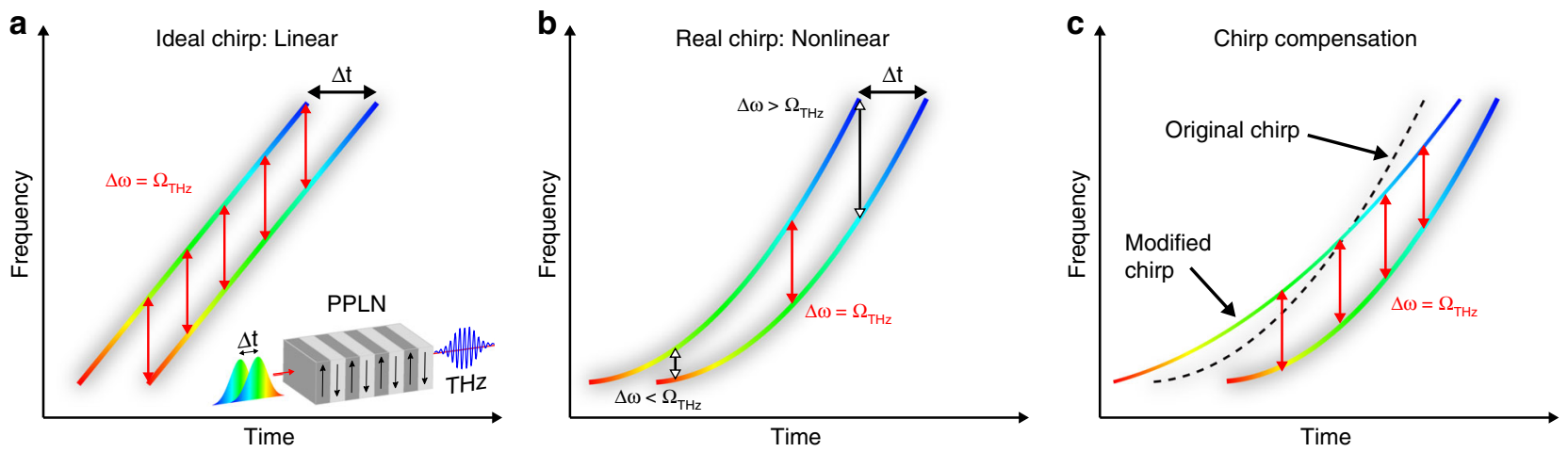

Fig. 1 Chirp-and-delay concept. a Two broadband but linearly chirped and delayed pulses provide narrow spectral features with constant instantaneous difference frequency $\Delta \omega$, which is converted in a PPLN crystal into a THz pulse of frequency $\Omega_{\mathrm{THz}}=\Delta \omega$. $\mathbf{b}$ Due to higher order dispersion $\Delta \omega$ varies along the pulse and limits the range where the provided difference frequency fulfils the phase matching condition in the PPLN. c Slightly tilting one of the pulses in time-frequency space regularises $\Delta \omega$ and thereby maximises $\mathrm{THz}$ generation

acceptance bandwidth of the PPLN around $\Omega_{\mathrm{THz}}$ over the full duration of the pulse, optimising the efficiency of the process.

Equivalently, this optimisation of the $\mathrm{THz}$ conversion efficiency can be described in the time domain: when the periodicity of the optical intensity modulation matches the $\mathrm{THz}$ carrier period, the $\mathrm{THz}$ generated by successive pulses in the train add coherently. Irregularities in the pulse-train periodicity induce destructive interferences which become more severe as the number of $\mathrm{THz}$ cycles increases. Monochromatising the difference frequency $\Delta \omega$, which determines the temporal beat frequency, ensures a regular pulse train and coherent addition of $\mathrm{THz}$ waves.

Millijoule-scale narrowband $\mathrm{THz}$ generation. To demonstrate this concept experimentally, we use a modified Mach-Zehnder interferometer (shown in Fig. 2a) in which uncompressed pulses from a Ti:Sapphire system are split into two identical copies and recombined after spectral phase manipulation.

Typical CPA systems have a positive chirp curvature, so monochromatising $\Delta \omega$ requires adding positive GDD and negative TOD (see Methods). However, negative TOD is generally not available. While it is conceptually simpler to apply dispersion compensation to only one pulse, as illustrated in Fig. 1, an equivalent solution is to add GDD to one pulse and TOD with reversed sign to the other. We therefore split the dispersion compensation between the two interferometer arms, which allows us to use custom-designed dispersion compensating mirrors (DCMs), available with positive TOD, to tune the second pulse. This design also compactifies the setup. A tunable amount of GDD is added by inserting a variable thickness of highdispersion glass (SF11) and TOD is added incrementally by replacing high-reflectors with DCMs.

In order to maximise the pump energy that we can apply to the DFG process, we employ large-aperture (shown in Fig. 2b), magnesium-doped PPLN (LA-PPMgLN) crystals which were custom fabricated by the Institute for Molecular Science in $J^{J a p a n}{ }^{29}$. Since Mach-Zehnder interferometers necessarily provide two identical outputs, we send each onto independent, cryogenically cooled crystals. This configuration provides a natural way to effectively double the crystal aperture, further increasing the allowable incident energy and making use of energy that would otherwise be wasted. Total optical pulse energies in excess of 1 Joule are thus safely applied to the crystals. The resulting $\mathrm{THz}$ pulse energy is then measured with a pyroelectric detector and the spectrum is characterised using a longitudinal interferometer.

Here we investigate the performance of crystals with a poling period of $330 \mu \mathrm{m}$ and a corresponding emission frequency of
$361 \mathrm{GHz}$, which is ideally suited for powering a THz-based accelerator $^{16}$. Details of the performance of a PPLN crystal designed for a higher frequency of $558 \mathrm{GHz}$ (i.e., with a periodicity of $212 \mu \mathrm{m}$ ), are presented in Supplementary Note 1 . For our driver laser, which has a GDD of $2.05 \times 10^{6} \mathrm{fs}^{2}$ and a TOD of $-4 \times 10^{6} \mathrm{fs}^{3}$, we calculate (see Methods) an optimum compensation of $\Delta \mathrm{GDD}=9100 \mathrm{fs}^{2}$ and $\Delta \mathrm{TOD}=54,000 \mathrm{fs}^{3}$ for the $330 \mu \mathrm{m}$ crystal. Note that these phase compensation amounts are much smaller than the original spectral phase (i.e., $\triangle \mathrm{GDD} \ll \mathrm{GDD}$ and $\triangle \mathrm{TOD} \ll \mathrm{TOD})$ which ensures the pulse duration is not significantly affected and is a key factor in the practicality of our technique.

Figure 3a shows the $\mathrm{THz}$ signal as a function of the delay between the driver pulses. In the uncompensated case (red dots), $\mathrm{THz}$ is generated with low signal levels over a broad range of delays. Here, each delay providing $\mathrm{THz}$ signal corresponds to matching of the instantaneous frequency difference, $\Delta \omega$, with the PPLN design frequency, $\Omega_{\mathrm{THz}}$, at a temporally and spectrally distinct part of the pulse. Adding GDD by increasing the amount of SF11 in the beam enhances the $\mathrm{THz}$ signal (blue triangles) by allowing more parts of the pulse to contribute to $\mathrm{THz}$ generation simultaneously. As a result, the range of $\mathrm{THz}$-generating delays narrows until most of the pulse is engaged at a single delay. The experimentally determined optimum (green squares) of $\triangle \mathrm{GDD}$ $=9200 \mathrm{fs}^{2}$ (or $49 \mathrm{~mm} \mathrm{SF11}$ ) is in very good agreement with our calculation and results in an 11-fold increase in $\mathrm{THz}$ output.

Using DCMs to tune the relative TOD between the two driver pulses, the $\mathrm{THz}$ signal is further enhanced by $20 \%$ yielding in total a 13-fold increase in output compared to the initial configuration (Fig. 3b). The measured optimum of $\triangle \mathrm{TOD}=$ $20,000 \mathrm{fs}^{3}$ is different from the predicted optimum, which may be attributable to the presence of fourth and higher orders in the pump spectral phase. In contrast to the GDD correction, the discrete nature of the TOD compensation makes fine-tuning of the TOD difficult.

The above optimisation of the THz yield can be understood as an optimisation of the temporal range over which the timedependent frequency difference $\Delta \omega(t)$ falls within the $\mathrm{THz}$ bandwidth $\Omega_{\mathrm{THz}} \pm \Delta \Omega_{\mathrm{THz}}$ accepted by the phase-matching process (Fig. 4a). The portion of the optical pulse contributing to $\mathrm{THz}$ generation is then simply estimated as the fraction satisfying $\left|\Delta \omega(t)-\Omega_{\mathrm{THz}}\right| \leq \Delta \Omega_{\mathrm{THz}}$. More precisely, this fraction is the integrated product of the phase-matching efficiency curve in the time domain and the temporal intensity profile of the optical pulse (Fig. 4a). This quantity serves as a good measure predicting the variation in $\mathrm{THz}$ output with delay and with GDD and TOD corrections, such as the enhancement of $\mathrm{THz}$ at 


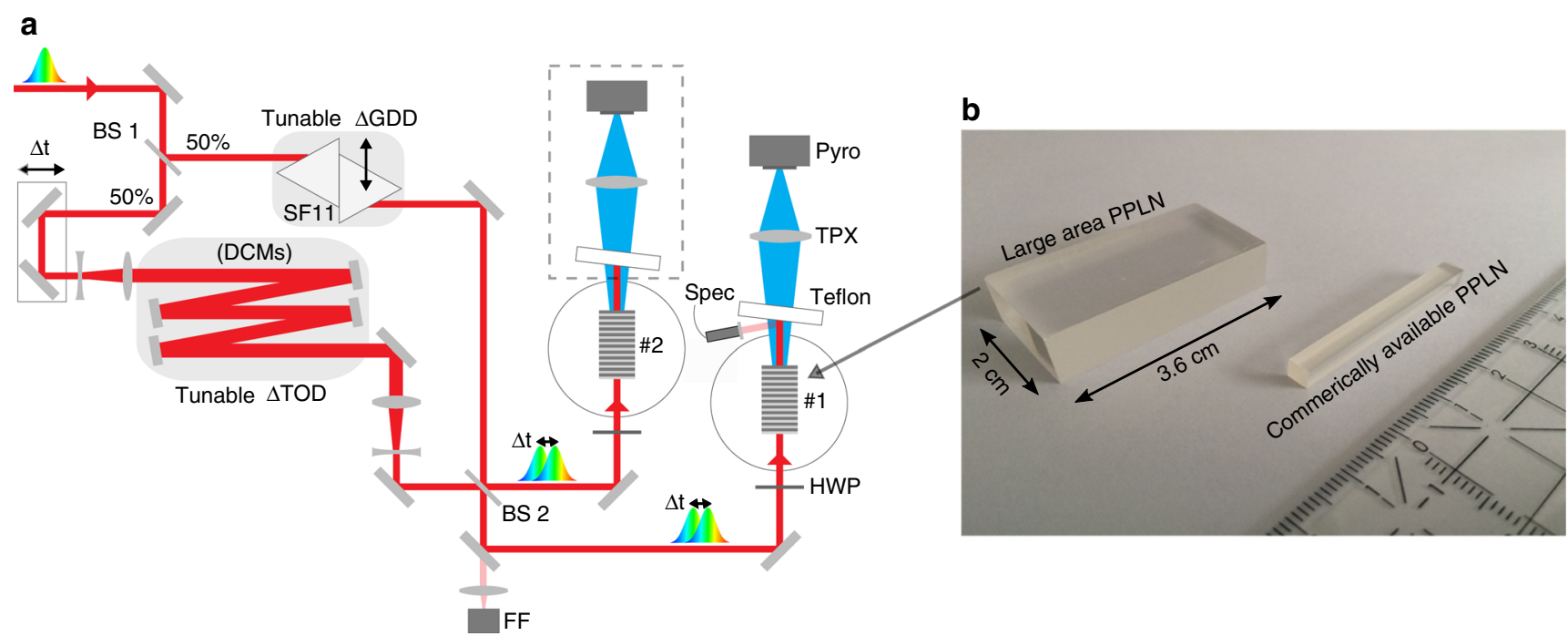

Fig. 2 Narrowband THz setup. a The driver laser output is split into two identical copies, whose GDD and TOD are independently tuned using a variable amount of high-dispersive SF11 glass (adding GDD), and a set of dispersion compensating mirrors (DCM), each adding a discrete amount of TOD. The recombined pulses generate narrowband THz pulses in large-aperture cryogenically cooled magnesium-doped PPLN crystals, shown in the photograph (b). To characterise the $\mathrm{THz}$ pulses, a pyroelectric detector is used either directly (pulse energy) or as the detector in a longitudinal interferometer (frequency). BS: beam splitter; FF: far field camera; HWP: half-wave plate

a

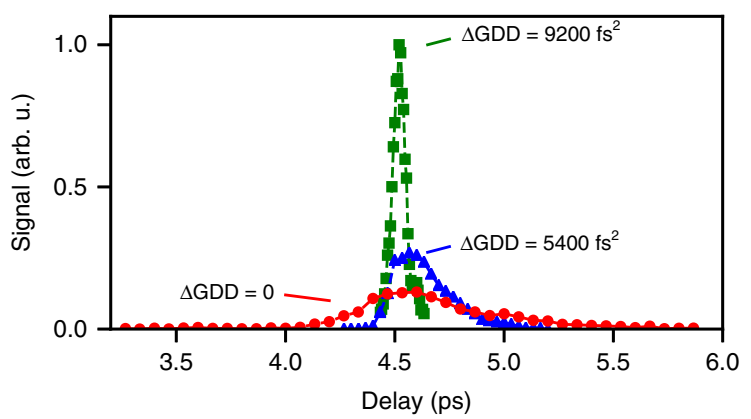

b

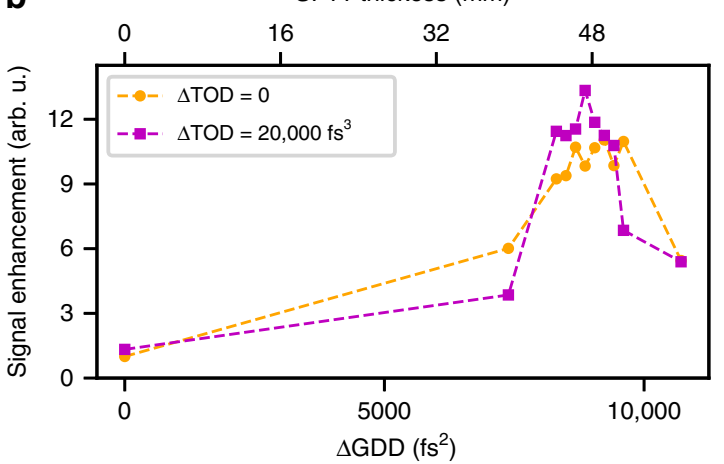

Fig. 3 Optimisation of the relative spectral phase. a Scan of pulse delay to match $\Delta \omega$ to the PPLN frequency $\Omega_{\mathrm{THz}}$. Adding $\Delta \mathrm{GDD}$, by inserting SF11 into one of the interferometer arms, the THz signal increases up to a factor of 11 (green squares), and the range of THz-generating delays narrows. $\mathbf{b}$ Conversion efficiency enhancement vs $\Delta$ GDD for varying amounts of $\Delta T O D$. For an optimum $\Delta T O D$, the signal increases by another $20 \%$ relative to no $\Delta T O D$ correction, yielding a total increase in $\mathrm{THz}$ output by a factor of 13

positive delays and the reduction of $\mathrm{THz}$ at negative delays (Fig. 4b).

To characterise the performance of the crystal, we use two definitions of conversion efficiency which highlight different quantities. The "extracted" efficiency, plotted in Fig. 5a, represents the ratio of useful $\mathrm{THz}$ energy extracted from the crystal to the driver incident energy. The peak extracted efficiency of $0.15 \%$ is achieved at a fluence of $180 \mathrm{~mJ} / \mathrm{cm}^{2}$. This figure corresponds to an "internal" efficiency of $0.24 \%$, which represents the ratio of $\mathrm{THz}$ and optical energies within the crystal. The internal efficiency serves as a useful metric for the optimisation of the DFG process independent from the practical complexities of input and output coupling. The fine-tuning of the TOD not only increases the conversion efficiency, but also increases the fluence at which optimum conversion efficiency occurs, leading to large increases in the total $\mathrm{THz}$ yield.

Figure $5 \mathrm{~b}$ shows the corresponding extracted $\mathrm{THz}$ energies. The highest individual $\mathrm{THz}$ pulse energy of $458 \mu \mathrm{J}$ and the highest average 2-crystal output of $0.6 \mathrm{~mJ}$ are achieved at a fluence of $350 \mathrm{~mJ} / \mathrm{cm}^{2}$. The uniform red-shift of the optical spectrum (inset) shows that pump depletion has occurred across the entire spectrum, which confirms that the full optical pulse engages in the DFG process. The spectrum of the generated radiation is measured using an interferometric autocorrelation of the $\mathrm{THz}$ pulse (Fig. $5 \mathrm{c}$ ). The Fourier transform of this trace shows a central frequency of $361 \mathrm{GHz}$ and confirms a sub-percent bandwidth (Fig. 5d). If the pulses were to be combined, the $0.6 \mathrm{~mJ}$ and roughly 200 ps pulses could produce a field strength of $\sim 18 \mathrm{MV}$ / $\mathrm{m}$ when focused to a $1 / \mathrm{e}^{2}$ waist of $2 \mathrm{~mm}\left(2.4 \times \lambda_{\mathrm{THz}}\right)$.

Note that the pump fluence for peak yield is well above the fluence for peak conversion efficiency, which suggests benefits from even larger aperture crystals. The difference in performance of the two crystals (Fig. 5b) is partly due to anti-reflection measures, applied to only one crystal, that reduce Fresnel losses and improve the extraction efficiency by 35\% (see Methods).

Our measurements show that the need for spectral-phase tuning is driven by the narrow acceptance bandwidth of the process. For a similar degree of tuning, we therefore expect a lower conversion efficiency for a crystal with a narrower phasematching bandwidth. In fact, this effect is confirmed by the lower 

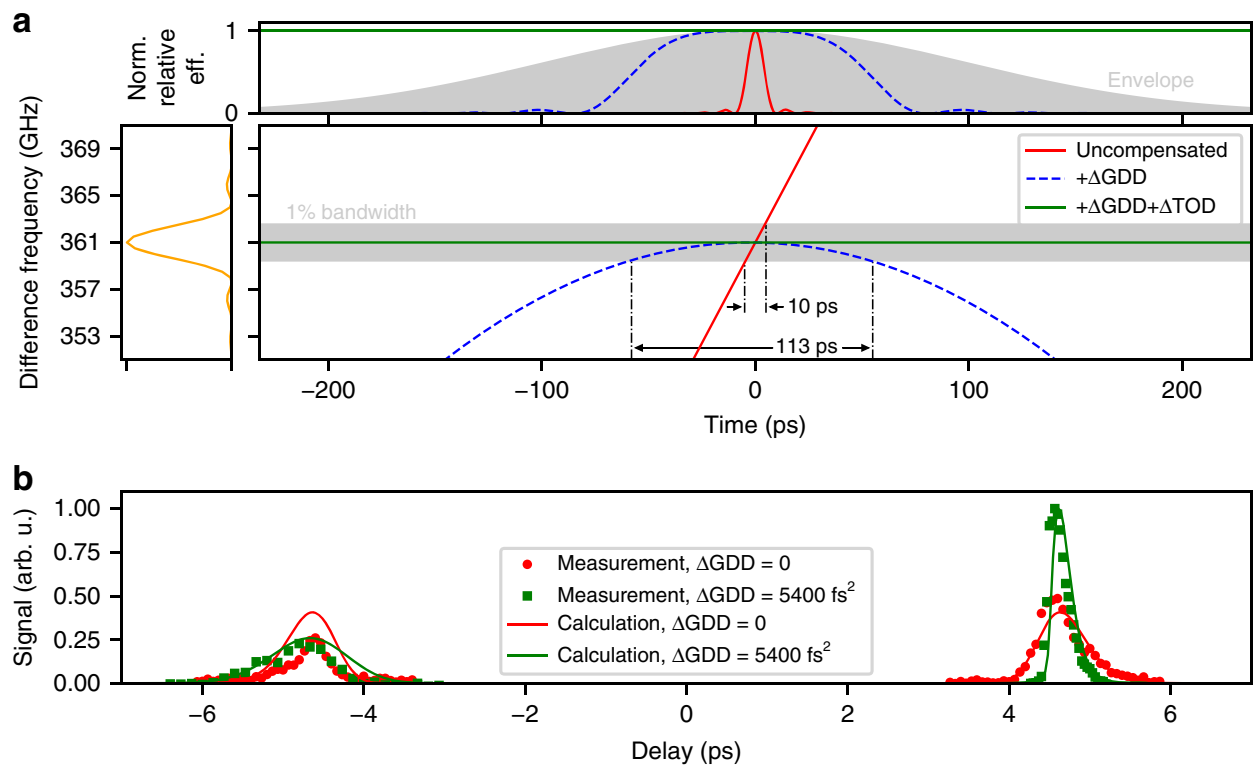

Fig. 4 Asymmetric chirp compensation. a The $330 \mu \mathrm{m}$ poling period PPLN has a central frequency of $\Omega_{\mathrm{THz}}=361 \mathrm{GHz}$ with a $1 \%$ bandwidth. Initially, the difference frequency $\Delta \omega$ varies linearly in time (red). As spectral phase compensation is applied adding $\Delta$ GDD (dashed blue) and further adding $\Delta T O D$ (solid green) the fraction of the pulse falling within the THz bandwidth $\Omega_{\mathrm{THz}} \pm \Delta \Omega_{\mathrm{THz}}$ and thus the generated THz energy, increases. The top inset shows the PPLN bandwidth multiplied by the difference frequency. The integrated product of this phase-matching efficiency with the pulse envelope (grey) is a measure for the fraction of the pulse contributing to THz generation. $\mathbf{b}$ Adding $\Delta \mathrm{GDD}$ (green) creates an asymmetry between the two pulses in time that reduces the $\mathrm{THz}$ signal at negative delays and enhances the $\mathrm{THz}$ at positive delays. For identical pulses (red) the $\mathrm{THz}$ signal is equal for positive and negative delays. A calculation (solid lines) based on the concept discussed in the main text and illustrated in panel a predicts the THz output in good agreement with the measurements (squares, dots)
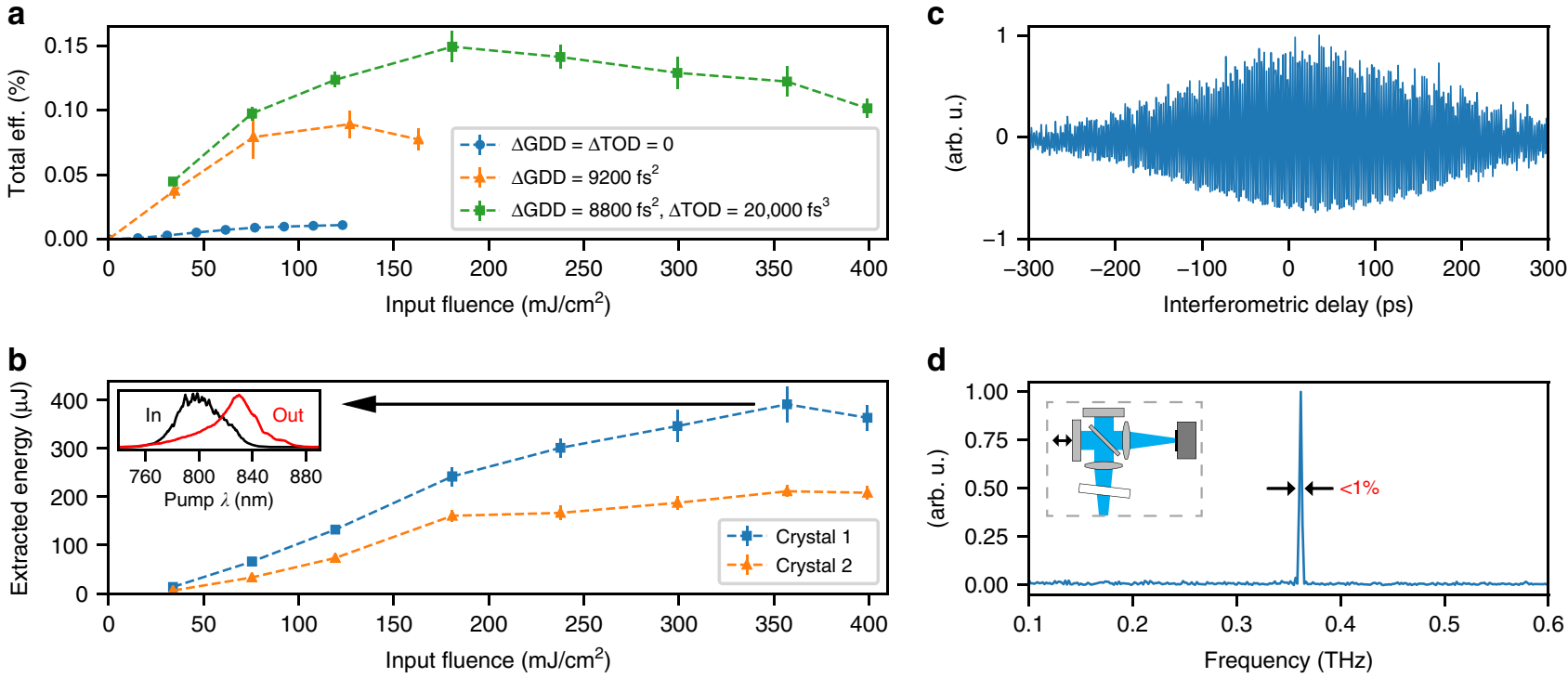

Fig. 5 High THz energy. a Fine tuning the spectral phase increases the fluence at which the maximum conversion efficiency occurs and reaches $0.15 \%$ at $180 \mathrm{~mJ} / \mathrm{cm}^{2}$ for crystal \#1. Note, that with simultaneous GDD and TOD correction, the optimum determined $\Delta$ GDD is slightly different than the value reported in Fig. 3a. b Using GDD and TOD compensation, an input fluence of $350 \mathrm{~mJ} / \mathrm{cm}^{2}$ generates individual THz pulse energies up to $458 \mu \mathrm{J}(392 \mu \mathrm{J}$ average) and $232 \mu \mathrm{J}(212 \mu \mathrm{J}$ average) in crystal \#1 and \#2, respectively. The difference in THz output is attributed to differences in the preparation of the crystal surfaces (see Methods). Significant red-shift of the driver spectrum is observed for high-energy THz generation indicating a high degree of optical cascading. c Interferometric correlation of the generated THz pulses and its frequency spectrum (d), confirming the central frequency of $361 \mathrm{GHz}$ and a sub-1\% bandwidth. The interferometer (inset) replaces the energy measurement setup, Fig. 2a. Note in c that each data point is averaged over 10 shots. The sampling of the frequency, however, accentuates the visual effect of the shot-to-shot fluctuations, while indeed the centre frequency is captured with high precision (see Supplementary Note 2). Error bars correspond to the standard deviation of 20 measurements in the case of $\mathbf{a}$ and $\mathbf{b}$ 
efficiency measured with the $212 \mu \mathrm{m}$ periodicity crystal which has a larger number of poling cycles and therefore a narrower acceptance bandwidth. Accounting for variations in absorption coefficient, crystal length and poling period, we predict a conversion efficiency of $0.08 \%$ at $558 \mathrm{GHz}$ which is in very good agreement with the measured value of $0.07 \%$ (see Supplementary Note 1).

Despite the large efficiency enhancements obtained by the relative spectral phase tuning, the maximum absolute conversion efficiency reported here is only twice larger than that reported by us previously ${ }^{27}$. An exact comparison between these results is not possible, however, since nonlinear crystals of different aperture, length and vendor were used.

In general, a proper comparison of efficiencies across different experiments requires a careful evaluation of the effects of differences in the laser, $\mathrm{THz}$ and crystal parameters, which can be very complex (see Supplementary Note 3). For example, the DFG efficiency intrinsically scales quadratically with $\mathrm{THz}$ frequency, due to higher $\mathrm{THz}$ photon energies, so one can expect higher efficiencies for higher frequencies. On the other hand, $\mathrm{THz}$ absorption also increases with frequency, mitigating, to varying degrees, the photon-energy effect. Differences in the crystal length, poling period, aperture and crystal manufacturer all also strongly affect the conversion process. The effect of crystal length on efficiency is especially complex, since length governs not only the $\mathrm{THz}$ bandwidth and therefore the sensitivity of the conversion process to TOD, but also the growth of cascading ${ }^{17}$ and amounts of pump depletion and $\mathrm{THz}$ reabsorption. Differences in the crystal aperture can lead to significant variations in efficiency via inhomogeneities in the poling structure ${ }^{29}$. The effects of crystal material and poling quality on multicycle $\mathrm{THz}$ generation are so far nearly unexplored. A detailed characterisation of these effects is thus an important part of the further optimisation of the multicycle $\mathrm{THz}$ generation process.

What we unambiguously demonstrate here is the role of TOD in detuning narrowband $\mathrm{THz}$ generation using the chirp-anddelay setup. The degree of relative enhancement achievable by tuning the relative spectral phase is robustly predictable from the amount of TOD and the phase-matching bandwidth, independent of other factors which may affect the efficiency. Mitigation of the effects of TOD is thus a required ingredient for optimising narrowband $\mathrm{THz}$ generation using chirp-and-delay.

\section{Discussion}

We demonstrate, for the first time, the sensitivity of narrowband $\mathrm{THz}$ generation to TOD in the chirp-and-delay setup and show that this sensitivity can be attributed to temporal variations in the difference-frequency content which cause a very strict phasematching condition not to be satisfied for the majority of the optical pulse. The effect of TOD can be understood in the time domain as inducing pulse-train irregularities which cause a destructive superposition of $\mathrm{THz}$ waves generated by the various pulses within the train. To address this issue, we introduce a powerful new technique based on relative spectral-phase tuning of the chirped pulses. The technique provides, for the first time, a simple and easily-implemented method to control and fine tune the temporal dependence of the difference-frequency spectrum, and hence the pulse-train periodicity, without changes to the laser architecture or to the pulse-train intensity and duration.

For high-energy narrowband $\mathrm{THz}$ generation, where the priority is optimising the optical-to- $\mathrm{THz}$ conversion efficiency, we adjust the spectral phase to monochromatise the differencefrequency spectrum and to create a regularised pulse-train (see Supplementary Notes 4 and 5), and thus obtain a 13-fold enhancement in $\mathrm{THz}$ yield (at $361 \mathrm{GHz}$ ). By using a Joule-class laser as the chirped-pulse source to pump two custom, largeaperture PPLN crystals (LA-PPMgLN ${ }^{29}$ ), we achieve a record energy of $0.6 \mathrm{~mJ}$ which exceeds previous results in optical generation of multicycle $\mathrm{THz}$ by over an order of magnitude. The internal conversion efficiency we measure of $0.24 \%$ is also nearly twice larger than previously reported for narrowband sources $^{20,26,27}$. This result is especially significant in light of the phase-matching difficulties associated with sub-percent $\mathrm{THz}$ bandwidths and the additional complexities associated with scaling to centimetre apertures and Joule-level pump energies ${ }^{29}$.

Millijoule-scale narrowband $\mathrm{THz}$ pulses represent a breakthrough which demonstrates the feasibility of the emerging technology of THz-based acceleration that holds high promise for revolutionising electron accelerators and the light sources based on them ${ }^{16}$. It is expected that $0.6 \mathrm{~mJ}$ of $\mathrm{THz}$ in a 200 -ps-long pulse as produced in this work could create a field strength of $50 \mathrm{MV} / \mathrm{m}$ when coupled in to a waveguide designed for matched acceleration. With acceleration over the roughly $4 \mathrm{~cm}$ allowed by such a pulse, sub-relativistic electrons could be accelerated beyond $2 \mathrm{MeV}$, an energy already relevant for ultrafast electron diffraction. This can be compared to past acceleration in a waveguide with broadband $\mathrm{THz}$, where the acceleration length was limited to $3 \mathrm{~mm}$ and the energy gain was limited to $7 \mathrm{keV}^{1}$.

However, our results are equally applicable for enhancing the yield of narrowband $\mathrm{THz}$ pulses at any energy level. For the average researcher with a $\mathrm{mJ}$ - or sub $\mathrm{mJ}$-scale laser system, making every photon count is an imperative. Order-ofmagnitude-scale efficiency enhancements are thus a game changer for enabling experiments requiring high-field, narrowband $\mathrm{THz}$ pulses, such as pumping of Josephson plasmon resonances in layered superconductors ${ }^{30-32}$. Spectroscopic applications may be accessible with our source parameters ${ }^{9}$, and we have demonstrated the technique at multiple frequencies. However, the lack of demonstrated tunability of the PPLN technology may limit the practical application to spectroscopic scenarios that do not require a large number of frequencies.

As chirp-and-delay is ubiquitously used for generating pulse trains, tuning the pulse-train is also relevant for a wide range of applications that require resonant excitation, including excitation of plasma waves ${ }^{33}$, high-order harmonic generation ${ }^{22}$, laser modulation of electron beams ${ }^{23}$, coherent anti-Stokes Raman scattering microscopy of biological structures ${ }^{24}$, excitation of atoms ${ }^{34}$, molecules and solids and driving photocathode injectors for conventional accelerators ${ }^{35}$. These applications have so far been limited by the presence of high-order phase in the chirpedpulse drivers and have suffered from the lack of practical possibilities to remove it. Our technique addresses this need. In addition, the technique opens up the possibility of generating pulse trains with customised temporal periodicity profiles with novel applications such as generation of pre-chirped multicycle $\mathrm{THz}$ pulses that compress under the action of waveguide dispersion, and thus address a difficult problem in the delivery of high-field $\mathrm{THz}$ to an interaction point ${ }^{36}$

\section{Methods}

Laser system. We use a 260-ps FWHM uncompressed, high-power CPA-based Ti: Sapphire laser providing $1.2 \mathrm{~J}$ at $5 \mathrm{~Hz}$ repetition rate. Inherent to the Öffner stretcher design, the laser pulses have a GDD of $\phi_{2}=2.05 \times 10^{6} \mathrm{fs}^{2}$ and a TOD of $\phi_{3}=-4 \times 10^{6} \mathrm{fs}^{3}$ with additional uncharacterised fourth and higher order spectral phase. The beam has a super-Gaussian profile of $13 \mathrm{~mm} 1 / \mathrm{e}^{2}$ intensity diameter, and a spectral width of more than $30 \mathrm{~nm}$ FWHM centred at $800 \mathrm{~nm}$.

Dispersion management. Using the common description of spectral phase, the time-dependent frequency of a chirped pulse can be written as $\omega\left(t, \phi_{2}, \phi_{3}\right)=\omega_{0}+$ $1 / \phi_{2} \times t-\phi_{3} / 2 \phi_{2}{ }^{3} \times t^{2}$, with $\omega_{0}$ the central frequency, and $\phi_{2}$ and $\phi_{3}$ the pulse GDD and TOD. Here, we neglect higher orders and assume the GDD is large. The parameters $\phi_{2}$ and $\phi_{3}$ are properties of the pulse and are typically inherent to the 
laser design. When there is no TOD (i.e., $\phi_{3}=0$ ), the chirp is linear and the instantaneous difference in frequency $\Delta \omega$ is constant, determined only by the chirp rate (i.e., GDD, $\phi_{2}$ ) and the delay:

$$
\Delta \omega(t)=\omega\left(t, \phi_{2}\right)-\omega\left(t-\Delta t, \phi_{2}\right)=\frac{\Delta t}{\phi_{2}} .
$$

In the presence of TOD, however, the chirp becomes nonlinear (i.e., curved), and $\Delta \omega$ becomes a linear function of time with a slope that is proportional to the TOD:

$$
\Delta \omega(t)=\omega\left(t, \phi_{2}, \phi_{3}\right)-\omega\left(t-\Delta t, \phi_{2}, \phi_{3}\right)=\left[\frac{\Delta t}{\phi_{2}}+\frac{\phi_{3}}{2 \phi_{2}^{3}} \Delta t^{2}\right]-\left[\frac{\phi_{3}}{\phi_{2}^{3}} \Delta \mathrm{t}\right] \times t .
$$

Applying the condition $\Delta \omega=\Omega_{\mathrm{THz}}$ shows that phase matching is only achieved at a particular time, $t^{*}$, which is dependent on the delay $\Delta t$ and given by:

$$
t^{*}(\Delta t)=\frac{\phi_{2}^{2}}{\phi_{3}}+\frac{1}{2} \Delta t-\frac{\phi_{2}^{3}}{\phi_{3} \Delta t} \Omega_{T H z}
$$

In the case of two identical chirped pulses, perfect phase matching requires elimination of the time-dependence of $\Delta \omega$, and thus can only be achieved by complete elimination of the TOD. Adjusting the relative dispersion of the two pulses, however, provides additional options. Adding a small amount of GDD, $\Delta \phi_{2}$, and TOD, $\Delta \phi_{3}$, reduces the curvature of the time-frequency mapping, which is equivalent to slightly tilting the pulse in time-frequency space, shown in Fig. 1c. The instantaneous difference in frequency between two chirped pulses, where one has been delayed by $\Delta t$ and tuned in spectral phase by adding $\Delta \phi_{2}$ and $\Delta \phi_{3}$, then reads

$$
\Delta \omega(t)=\omega\left(t, \phi_{2}, \phi_{3}\right)-\omega\left(t-\Delta t, \phi_{2}+\Delta \phi_{2}, \phi_{3}+\Delta \phi_{3}\right) .
$$

It is straightforward to show that $\Delta \omega(t)$ is now a second order equation in $t$, expressible as $\Delta \omega(t)=c_{0}+c_{1} \times t+c_{2} \times t^{2}$ with $c_{i}=c_{i}\left(\phi_{2}, \phi_{3}, \Delta \phi_{2}, \Delta \phi_{3}, \Delta t\right)$. More explicitly stated,

$$
\begin{aligned}
\Delta \omega(t)= & {\left[\frac{1}{\phi_{2}+\Delta \phi_{2}} \Delta t+\frac{\phi_{3}+\Delta \phi_{3}}{2\left(\phi_{2}+\Delta \phi_{2}\right)} \Delta t^{2}\right] } \\
& +\left[\frac{1}{\phi_{2}}-\frac{1}{\phi_{2}+\Delta \phi_{2}}-\frac{\phi_{3}+\Delta \phi_{3}}{\left(\phi_{2}+\Delta \phi_{2}\right)^{3}} \Delta t\right] \times t \\
& -\left[\frac{\phi_{3}}{2 \phi_{2}^{3}}-\frac{\phi_{3}+\Delta \phi_{3}}{2\left(\phi_{2}+\Delta \phi_{2}\right)^{3}}\right] \times t^{2} .
\end{aligned}
$$

According to this expression, a constant instantaneous frequency difference $\Delta \omega=$ $\Omega_{\mathrm{THz}}$, as illustrated in Fig. $1 \mathrm{c}$, can be recovered by setting the coefficients $c_{1}$ and $c_{2}$ to zero, and solving for $\Delta \phi_{2}, \Delta \phi_{3}$, and $\Delta t$

$$
\begin{aligned}
& \Delta \phi_{2}=\phi_{2}\left(\frac{\phi_{2}}{\phi_{2}-2 \phi_{3} \Omega_{T H z}}\right)^{\frac{1}{2}}-\phi_{2} \\
& \Delta \phi_{3}=\phi_{3}\left(\frac{\phi_{2}}{\phi_{2}-2 \phi_{3} \Omega_{T H z}}\right)^{\frac{3}{2}}-\phi_{3} \\
& \Delta t=\frac{2 \phi_{2} \Omega_{T H z}}{1+\left(1-\frac{2 \phi_{3} \Omega_{T H z}}{\phi_{2}}\right)^{\frac{1}{2}}} .
\end{aligned}
$$

THz generation. The chirp-and- delay concept is implemented using a MachZehnder interferometer, with the GDD and TOD compensation distributed between the two interferometer arms. SF11 adds GDD of $\Delta \phi_{2} / L=+187.5 \mathrm{fs}^{2} / \mathrm{mm}$ and additional small TOD of $\Delta \phi_{3} / L=+126 \mathrm{fs}^{3} / \mathrm{mm}$. Custom dielectric mirrors each add $\Delta \phi_{3}=+20,000 \mathrm{fs}^{3}$ and no additional GDD, independently confirmed with a white-light interferometer. For experiments at high fluence, two telescopes locally increase the beam size on the DCMs. The dispersion through the lenses is about $800 \mathrm{fs}^{2}$ and compensated with additional $4.4 \mathrm{~mm}$ SF11, which is not explicitly mentioned in the main text. The setup is very sensitive to the angular overlap of the two beams, which we ensure to sub-100 $\mu$ rad level with a far field camera after the second beam splitter. The PPLN crystals are cooled below $100 \mathrm{~K}$ in a cryostat operating at $8 \times 10^{-4} \mathrm{mbar}$. The entrance window is AR coated for the NIR driver pulses. A ceramic aperture in front of the crystals $(8.5 \times 14.5 \mathrm{~mm}$ crystal $\# 1,9.5 \times 14.5 \mathrm{~mm}$ crystal \#2) prevents the beam from clipping on the crystal edges. We use Teflon plates with calibrated transmission, to separate the driver IR beam from the generated $\mathrm{THz}$ pulses. The $\mathrm{THz}$ energy is detected with a pyroelectric sensor (Gentec-EO: THZ9B-BL-BNC) that had been calibrated to a device calibrated by the Physikalisch-Technische Bundesanstalt (PTB). Each data point reported in the figures is averaged over 5 pulses (long delay scan, Fig. 4b), 100 pulses (GDD and TOD tuning, Fig. 3), and 20 pulses (fluence scan, Fig. 5). When error bars are not shown the error is below $5 \%$ rms and not visible within the given figure.

PPLN crystals. We use custom $10 \times 15 \mathrm{~mm}^{2}$ large aperture crystals ${ }^{29}$ with a length of $36 \mathrm{~mm}$ and poling periods of $330 \mu \mathrm{m}$ and $212 \mu \mathrm{m}$, corresponding to $\mathrm{THz}$ pulses of $361 \mathrm{GHz}$ and $558 \mathrm{GHz}$, respectively. For the generation of high-energy $\mathrm{THz}$ pulses reported in Fig. 5, the $361 \mathrm{GHz}$ crystal \#1 is AR coated for the driver laser and features a $100-\mu \mathrm{m}$ fused silica wafer at the end facet of the crystal to minimise Fresnel losses and maximise THz extraction. For comparison, the $361 \mathrm{GHz}$ crystal
$\# 2$ is left uncoated and without a wafer installed. The AR coating and fused silica wafer together enhance the THz output of crystal \#1 by about 35\% compared to crystal \#2. The remaining $25 \%$ difference in observed $\mathrm{THz}$ output is attributed to variations in the crystal manufacturing.

\section{Data availability}

The data that support the findings of this study are available from the corresponding author upon reasonable request.

Received: 8 February 2019 Accepted: 21 May 2019

Published online: 13 June 2019

\section{References}

1. Nanni, E. A. et al. Terahertz-driven linear electron acceleration. Nat. Commun. 6, 8486 (2015).

2. Huang, W. R. et al. Terahertz-driven, all-optical electron gun. Optica 3 , 1209-1212 (2016).

3. Walsh, D. A. et al. Demonstration of sub-luminal propagation of single-cycle terahertz pulses for particle acceleration. Nat. Commun. 8, 421 (2017).

4. Kealhofer, C. et al. All-optical control and metrology of electron pulses. Science 352, 429-433 (2016).

5. Frühling, U. et al. Single-shot terahertz-field-driven X-ray streak camera. Nat Photon. 3, 523-528 (2009).

6. Grguraš, I. et al. Ultrafast X-ray pulse characterization at free-electron lasers Nat. Photon. 6, 852-857 (2012)

7. Dienst, A. et al. Bi-directional ultrafast electric-field gating of interlayer charge transport in a cuprate superconductor. Nat. Photon. 5, 485-488 (2011).

8. Först, M. et al. Nonlinear phononics as an ultrafast route to lattice control. Nat. Phys. 7, 854-856 (2011).

9. Hebling, J., Yeh, K.-L., Hoffmann, M. C. \& Nelson, K. A. High-power THz generation, $\mathrm{THz}$ nonlinear optics, and $\mathrm{THz}$ nonlinear spectroscopy. IEEE J. Sel. Top. Quantum Electron. 14, 345-353 (2008).

10. Hirori, H., Doi, A., Blanchard, F. \& Tanaka, K. Single-cycle terahertz pulses with amplitudes exceeding $1 \mathrm{MV} / \mathrm{cm}$ generated by optical rectification in $\mathrm{LiNbO}_{3}$. Appl. Phys. Lett. 98, 091106 (2011)

11. Hoffmann, M. C. et al. Coherent single-cycle pulses with $\mathrm{MV} / \mathrm{cm}$ field strengths from a relativistic transition radiation light source. Opt. Lett. 36, 4473-4475 (2011)

12. Fülöp, J. A. et al. Efficient generation of $\mathrm{THz}$ pulses with $0.4 \mathrm{~mJ}$ energy. Opt. Express 22, 20155-20163 (2014).

13. Vicario, C., Monoszlai, B. \& Hauri, C. P. GV/m single-cycle Terahertz fields from a laser-driven large-size partitioned organic crystal. Phys. Rev. Lett. 112 213901 (2014)

14. Wong, L. J., Fallahi, A. \& Kärtner, F. X. Compact electron acceleration and bunch compression in $\mathrm{THz}$ waveguides. Opt. Express 21, 9792-9806 (2013).

15. Kampfrath, T., Tanaka, K. \& Nelson, K. A. Resonant and nonresonant control over matter and light by intense terahertz transients. Nat. Photon. 7, 680-690 (2013).

16. Kärtner, F. X. et al. AXSIS: Exploring the frontiers in attosecond X-ray science, imaging and spectroscopy. Nucl. Instrum. Methods Phys. Res. A 829, 24-29 (2016).

17. Ravi, K., Schimpf, D. N. \& Kärtner, F. X. Pulse sequences for efficient multicycle terahertz generation in periodically poled lithium niobate. Opt. Express 24, 25582-25607 (2016)

18. Lee, Y.-S. et al. Generation of narrow-band terahertz radiation via optical rectification of femtosecond pulses in periodically poled lithium niobate. Appl. Phys. Lett. 76, 2505 (2000).

19. Vodopyanov, K. L. Optical generation of narrow-band terahertz packets in periodically-inverted electro-optic crystals: conversion efficiency and optimal laser pulse format. Opt. Express 14, 2263-2276 (2006).

20. Carbajo, S. et al. Efficient narrowband terahertz generation in cryogenically cooled periodically poled lithium niobate. Opt. Lett. 40, 5762-5765 (2015)

21. Weling, A. S., Hu, B. B., Froberg, N. M. \& Auston, D. H. Generation of tunable narrow-band $\mathrm{THz}$ radiation from large aperture photoconducting antennas. Appl. Phys. Lett. 64, 137 (1994).

22. O’Keeffe, K., Robinson, T. \& Hooker, S. M. Quasi-phase-matching high harmonic generation using trains of pulses produced using an array of birefringent plates. Opt. Express 20, 6236-6247 (2012).

23. Ungelenk, P. et al. Continuously tunable narrowband pulses in the $\mathrm{THz}$ gap from laser-modulated electron bunches in a storage ring. Phys. Rev. Accel. Beams 20, 020706 (2017).

24. Malinovskaya, S. A. \& Malinovsky, V. S. Chirped-pulse adiabatic control in coherent anti-Stokes Raman scattering for imaging of biological structure and dynamics. Opt. Lett. 32, 707-709 (2007) 
25. Weling, A. S. \& Auston, D. H. Novel sources and detectors for coherent tunable narrow-band terahertz radiation in free space. J. Opt. Soc. Am. B 13, 2783-2792 (1996).

26. Chen, Z., Zhou, X., Werley, C. A. \& Nelson, K. A. Generation of high power tunable multicycle terahertz pulses. Appl. Phys. Lett. 99, 071102 (2011).

27. Ahr, F. et al. Narrowband terahertz generation with chirped-and-delayed laser pulses in periodically poled lithium niobate. Opt. Lett. 42, 2118-2121 (2017).

28. Hellerer, T., Enejder, A. M. K. \& Zumbusch, A. Spectral focusing: High spectral resolution spectroscopy with broad-bandwidth laser pulses. Appl. Phys. Lett. 85, 25 (2004).

29. Ishizuki, H. \& Taira, T. Improvement of laser-beam distortion in largeaperture PPMgLN device by using X-axis Czochralski-grown crystal. Opt. Express 22, 19668-19673 (2014).

30. Savel'ev, S., Rakhmanov, A. L., Yampol'skii, V. A. \& Nori, F. Analogues of nonlinear optics using terahertz Josephson plasma waves in layered superconductors. Nat. Phys. 2, 521-525 (2006).

31. Laplace, Y. \& Cavalleri, A. Josephson plasmonics in layered superconductors. Adv. Phys. X 1, 387-411 (2016).

32. Dienst, A. et al. Optical excitation of Josephson plasma solitons in a cuprate superconductor. Nat. Mater. 12, 535-541 (2013).

33. Shalloo, R. J. et al. Generation of laser pulse trains for tests of multi-pulse laser wakefield acceleration. Nucl. Instrum. Methods Phys. Res. A 829, 383-385 (2016).

34. Temkin, R. J. Excitation of an atom by a train of short pulses. J. Opt. Soc. Am. B 10, 830-839 (1993).

35. Siders, C. W., Siders, J. L. W., Taylor, A. J., Park, S.-G. \& Weiner, A. M. Efficient high-energy pulse-train generation using a $2^{\mathrm{n}}$-pulse michelson interferometer. Appl. Opt. 37, 5302-5305 (1998).

36. Zhang, D. et al. Segmented terahertz electron accelerator and manipulator (STEAM). Nat. Photon. 12, 336-342 (2018).

\section{Acknowledgements}

We thank M. Schnepp for help with the laser system, M. Schust and T. Tilp for technical support on the experimental setup, P. Messner for assistance with the vacuum system, K. Ravi for theoretical discussions, and S.-H. Chia for help with the white-light interferometry measurements. We acknowledge support from the European Research Council ERC Grant (609920), BMBF grant 05K16GU2, PIER project PIF-2017-67, and the DESY Strategy Fund. S.W.J. and V.L. acknowledge support by the European Regional Development Fund (ERDF) (CZ.02.1.01/0.0/0.0/15_008/0000162). H.I. and T.T. acknowledge support from the JST-Mirai Program Grant Number JPMJMI17A1, Japan.

\section{Author contributions}

N.H.M. proposed the asymmetric compensation technique, with S.W.J. contributing to the development. S.W.J. developed the theoretical description of the concept. S.W.J. and F.A. conducted the experiments with assistance from V.L., T.E., and N.H.M.; S.W.J. and F.A. analysed the data with detailed input from N.H.M., A.R.M., and F.X.K.; A.L.C. facilitated the collaboration with H.I. and T.T., who provided the large aperture P.P.L.N crystals. S.W.J., N.H.M., and A.R.M. wrote the manuscript with feedback from all authors. A.R.M. and F.X.K. led the project.

\section{Additional information}

Supplementary Information accompanies this paper at https://doi.org/10.1038/s41467019-10657-4.

Competing interests: The authors declare no competing interests.

Reprints and permission information is available online at http://npg.nature.com/ reprintsandpermissions/

Journal peer review information: Nature Communications thanks János Hebling and the other anonymous reviewer for their contribution to the peer review of this work. Peer reviewer reports are available.

Publisher's note: Springer Nature remains neutral with regard to jurisdictional claims in published maps and institutional affiliations.

Open Access This article is licensed under a Creative Commons Attribution 4.0 International License, which permits use, sharing, adaptation, distribution and reproduction in any medium or format, as long as you give appropriate credit to the original author(s) and the source, provide a link to the Creative Commons license, and indicate if changes were made. The images or other third party material in this article are included in the article's Creative Commons license, unless indicated otherwise in a credit line to the material. If material is not included in the article's Creative Commons license and your intended use is not permitted by statutory regulation or exceeds the permitted use, you will need to obtain permission directly from the copyright holder. To view a copy of this license, visit http://creativecommons.org/ licenses/by/4.0/.

(C) The Author(s) 2019 